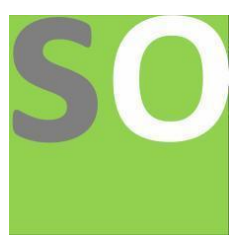

Article title: The strong cosmic censorship conjecture may be violated

Authors: Wen-Xiang Chen[1]

Affiliations: South China Normal University[1]

Orcid ids: 0000-0002-0560-8280[1]

Contact e-mail: 954763891@qq.com

License information: This work has been published open access under Creative Commons Attribution License http://creativecommons.org/licenses/by/4.0/, which permits unrestricted use, distribution, and reproduction in any medium, provided the original work is properly cited. Conditions, terms of use and publishing policy can be found at https://www.scienceopen.com/.

Preprint statement: This article is a preprint and has not been peer-reviewed, under consideration and submitted to ScienceOpen Preprints for open peer review.

DOI: 10.14293/S2199-1006.1.SOR-.PPILXSQ.v1

Preprint first posted online: 25 May 2020

Keywords: strong cosmic censorship conjecture, entropy reduction, superradiant stability 


\title{
The strong cosmic censorship conjecture may be violated
}

\author{
Wen-Xiang Chen* \\ Institute of quantum matter, \\ School of Physics and Telecommunication Engineering, \\ South China Normal University, \\ Guangzhou 510006, China
}

\begin{abstract}
Penrose's intense cosmic censorship conjecture asserts that the Cauchy horizon inside the dynamically formed black hole is unstable to the remaining material field that falls into the black hole. The physical importance of this conjecture stems from the fact that it provides the necessary conditions for general relativity to become truly deterministic gravity. In a recent paper by Hod, it provides a proof based on Beckenstein 's second law of generalized thermodynamics that confirms the validity of the interesting Penrose conjecture in the space-time of curved black holes. Recently, an article of mine obtained interesting results about the superradiant stability of Kerr black holes. The result contains some conclusions that violate the "no-hair theorem". We know that the phenomenon of black hole superradiation is a process of entropy reduction, and connecting Hod's paper with my paper, I found that the strong cosmic censorship conjecture may be violated.
\end{abstract}

Keywords: strong cosmic censorship conjecture, entropy reduction, superradiant stability

\section{INTRODUCTION}

The strong cosmic censorship conjecture asserts that general relativity, like classical mechanics, is a deterministic theory. In other words, the state of the deterministic system is doomed at the initial moment.In order to ensure the certainty of general relativity as a successful gravitational theory, Penrose proposed the cosmic censorship conjecture, according to which the mysterious "cosmic censorship" can prevent distant observers from being disturbed by highly curved space-time. This interesting conjecture concludes in particular that the Cauchy horizons in physically realistic (dynamically formed) black holes are singular, and these layers mark the boundary where the Einstein field equation loses its predictive power. If correct, the powerful form of Penrose 's conjecture would mean that physical observers are always confined to living in space and time, and general relativity is a physically successful and mathematically deterministic gravity theory. Hod[9] proved that internal black hole space-time cannot be extended beyond these horizons in a non-unique (uncertainty) manner and physically realistic (dynamically formed) black hole space-time respects Penrose's strong cosmic censorship conjecture.

In 1972, Press and Teukolsky[13] proposed that It is possible to add a mirror to the outside of a black hole to make a black hole bomb (according to the current explanation, this is a scattering process involving classical mechanics and quantum mechanics[3, $6,11,12])$.

When a bosonic wave is impinging upon a rotating black hole, the wave reflected by the event horizon will be

\footnotetext{
*Electronic address: wxchen4277@qq.com
} 
amplified if the wave frequency $\omega$ lies in the following superradiant regime[13, 15, 16]

$$
0<\omega<m \Omega_{H}, \Omega_{H}=\frac{a}{r_{+}^{2}+a^{2}},
$$

where $m$ is azimuthal number of the bosonic wave mode, $\Omega_{H}$ is the angular velocity of black hole horizon. This amplification is superradiant scattering. Therefore, through the superradiation process, the rotational energy of the black hole can be extracted. If there is a mirror between the black hole's horizon and infinite space, the amplified wave will scatter back and forth and grow exponentially, which will cause the black hole's superradiation to become unstable.

Since the physical behavior of the singularity is unknown, if the singularity can be observed by other parts of spacetime, then the causality will be broken, and physics may lose the ability to predict. According to Penrose-Hawkin's singularity theory, singularities are inevitable in the case of physical significance, which makes the above hypothesis (the existence of naked singularities will cause the causality to break) also inevitable.

In addition, if the naked singularity does not exist, then the universe will become deterministic-it is possible to depend only on the state of the universe at a certain moment (more precisely, a space-like three-dimensional hypersurface called the Cauchy surface State), inferring the entire evolution of the universe (perhaps it is necessary to exclude the limited space hidden in the horizon of the singular point). If the cosmological examination hypothesis fails, it will lead to the definite failure of the universe, because it is impossible to derive the space-time behavior of the universe from the causality of the singular point. The cosmic censorship hypothesis is a formal concern of the physics community. When referring to the event horizon of a black hole, some form of cosmological censorship hypothesis is always involved.

From [4][5], $\mu=y \omega$, we get inequality (2)

$$
\frac{m a}{\frac{2 r_{-}^{2}}{y}+\left(M r_{-}-r_{-}^{2}\right) y+\left(y+\frac{1}{y}\right) \frac{a^{2}}{2 M}\left(M-r_{-}\right)}<\mu<\sqrt{2} m \Omega_{H},
$$

where $\mu$ is the mass.

That result contained some conclusions that violated the strong cosmic censorship conjecture.

\section{DESCRIPTION OF THE KERR-BLACK-HOLE SYSTEM}

The metric of the Kerr black hole[8, 10] (in natural unit $\mathrm{G}=\mathrm{c}=1$ ) is

$$
\begin{gathered}
d s^{2}=-\frac{\Delta}{\rho^{2}}\left(d t-a \sin ^{2} \theta d \phi\right)^{2}+\frac{\rho^{2}}{\Delta} d r^{2}+\rho^{2} d \theta^{2}+\frac{\sin ^{2} \theta}{\rho^{2}}\left[a d t-\left(r^{2}+a^{2}\right) d \phi\right]^{2} . \\
\Delta=r^{2}-2 M r+a^{2}, \rho^{2}=r^{2}+a^{2} \cos ^{2} \theta .
\end{gathered}
$$

We know the Klein-Gordon equation

$$
\left(\nabla^{\nu} \nabla_{\nu}-\mu^{2}\right) \Psi=0
$$

Eigenvalues of the above formula and spherical harmonic functions can be written as

$$
\Psi_{\operatorname{lm}}(\mathrm{t}, \mathrm{r}, \theta, \phi)=\sum_{\mathrm{l}, \mathrm{m}} \mathrm{e}^{\mathrm{im} \phi} \mathrm{S}_{\operatorname{lm}}(\theta) \mathrm{R}_{\operatorname{lm}}(\mathrm{r}) \mathrm{e}^{-\mathrm{i} \omega \mathrm{t}}
$$


Substituting (6) into the Klein-Gordon wave equation, we get that the angular function $\mathrm{S}_{\operatorname{lm}}(\theta)$ satisfies the following equation $[1,7,14]$

$$
\frac{1}{\sin \theta} \frac{d\left(\sin \theta \frac{d S_{l m}}{d \theta}\right)}{d \theta}+\left[K_{l m}+a^{2}\left(\mu^{2}-\omega^{2}\right) \sin ^{2} \theta-\frac{m^{2}}{\sin ^{2} \theta}\right] S_{l m}=0 ;
$$

For the references[1, 7, 14],we know inequality(8)

$$
K_{l m} \geq m^{2}-a^{2}\left(\mu^{2}-\omega^{2}\right),
$$

where $l$ is the spherical harmonic index, $m$ is the azimuthal harmonic index with $-l \leq m \leq l$ and $\omega$ is the energy of the mode.

\section{KERR-BLACK-HOLE-MASSIVE-SCALAR-FIELD SYSTEM AND SUPERRADIANT QUANTUM EFFECT}

The radial Klein-Gordon equation[7, 14] obeyed by $R_{l m}$ is given by

$$
\Delta \frac{d\left(\Delta \frac{d R}{d r}\right)}{d r}+U R=0
$$

where

$$
\Delta=r^{2}-2 M r+a^{2}
$$

and

$$
U=\left[\omega\left(\mathrm{r}^{2}+a^{2}\right)-\mathrm{m} a\right]^{2}+\Delta\left[2 \mathrm{~m} a \omega-\mu^{2}\left(\mathrm{r}^{2}+a^{2}\right)-K_{l m}\right] .
$$

The inner and outer horizons of the black hole are

$$
r_{ \pm}=M \pm \sqrt{M^{2}-a^{2}}
$$

and we know that

$$
r_{+}+r_{-}=2 M, r_{+} r_{-}=a^{2} .
$$

We use tortoise coordinate $r_{*}$ by equation $\frac{d r_{*}^{2}}{d r^{2}}=\frac{r^{2}}{\Delta}$ and another radial function $\psi=$ rR.We get the following equation

$$
\frac{\mathrm{d}^{2} \psi}{\mathrm{dr}_{*}^{2}}+V \psi=0
$$

where

$$
V=\frac{U}{r^{4}}-\frac{2 \Delta}{r^{6}}\left(M r-a^{2}\right) .
$$

Then we get the asymptotic solutions for the radial wave equation below

$$
r \rightarrow \infty\left(\mathrm{r}_{*} \rightarrow \infty\right) \Rightarrow R_{\operatorname{lm}} \sim \frac{1}{r} e^{-\sqrt{\mu^{2}-\omega^{2}} r_{*}},
$$




$$
r \rightarrow r_{+}\left(r_{*} \rightarrow-\infty\right) \Rightarrow R_{\operatorname{lm}} \sim e^{-i\left(\omega-m \Omega_{H}\right) r_{*}}
$$

When

$$
\omega^{2}-\mu^{2}<0
$$

there is a bound state of the scalar field.

When $\varphi=\Delta^{\frac{1}{2}} R$, radial potential equation(9) can be transformed into the following equation

$$
\frac{d^{2} \varphi}{d r^{2}}+\left(\omega^{2}-V_{1}\right) \varphi=0, V_{1}=\omega^{2}-\frac{U+M^{2}-a^{2}}{\Delta^{2}}
$$

It was previously proved[4]that, the no-hair theorem is not necessarily true under the superradiation quantum effect. The previous literature[2] indicates that the superradiation effect is a process of entropy subtraction. The superradiant instability(The case under the no-hair theorem) of black holes is actually a phenomenon that occurs when the entropy reaches the maximum when the boson enters the black hole.

\section{THAT RESULT CONTAINED SOME CONCLUSIONS THAT VIOLATED THE STRONG COSMIC CENSORSHIP CONJECTURE}

For $\mu^{2}=y^{2} \omega^{2}$, when $y>4.352$, there exists a certain interval to let the inequality

$$
\frac{m a}{\frac{2 r_{-}^{2}}{y}+\left(M r_{-}-r_{-}^{2}\right) y+\left(y+\frac{1}{y}\right) \frac{a^{2}}{2 M}\left(M-r_{-}\right)}<\mu<\sqrt{2} m \Omega_{H},
$$

be satisfied. So the Kerr black hole is superradiantly stable at that time [5].

We see that when $y$ is very large, the superradiant stability interval of $\mu$ can always include some numerical solutions of the superradiant instability(The case under the no-hair theorem) obtained by previous people. For[4],at that time, the black hole entropy began to decrease.Hod[9] showed that the entropy of black holes remains constant even under scalar interference, so the strong cosmic censorship hypothesis holds. A recent article[4] showed that the entropy of a black hole can be reduced under superradiation.I found that the strong cosmic censorship conjecture may be violated.

\section{SUMMARY}

We know that the phenomenon of black hole superradiation is a process of entropy reduction, and connecting Hod's paper with my paper, I found that the strong cosmic censorship conjecture may be violated.

[1] Berti, E., Cardoso, V. and Casals, M. [2006], 'Eigenvalues and eigenfunctions of spin-weighted spheroidal harmonics in four and higher dimensions', Physical Review D 73(2), 024013.

[2] Brito, R., Cardoso, V. and Pani, P. [2015], 'Superradiance', Lect. Notes Phys 906(1), 1501-06570.

[3] Cardoso, V., Dias, O. J., Lemos, J. P. and Yoshida, S. [2004], 'Black-hole bomb and superradiant instabilities', Physical Review D 70(4), 044039.

[4] Chen, W.-X. [2020], 'The possibility of the no-hair theorem being violated', Available at SSRN 3569639. 
[5] Chen, W.-X. and Huang, Z.-Y. [2019], 'Superradiant stability of the kerr black hole', International Journal of Modern Physics D.

URL: https://doi.org/10.1142/S0218271820500091

[6] Greiner, W., Müller, B. and Rafelski, J. [1985], Evolution of the vacuum state in supercritical potentials, in 'Quantum Electrodynamics of Strong Fields', Springer, pp. 257-299.

[7] Hartman, T., Song, W. and Strominger, A. [2010], 'Holographic derivation of kerr-newman scattering amplitudes for general charge and spin', Journal of High Energy Physics 2010(3), 118.

[8] Herdeiro, C. A. and Radu, E. [2014], 'Kerr black holes with scalar hair', Physical review letters 112(22), 221101.

[9] Hod, S. [2020], 'A proof of the strong cosmic censorship conjecture', International Journal of Modern Physics D .

[10] Kerr, R. P. [1963], 'Gravitational field of a spinning mass as an example of algebraically special metrics', Physical review letters 11(5), 237.

[11] Manogue, C. A. [1988], 'The klein paradox and superradiance', Annals of Physics 181(2), 261-283.

[12] Penrose, R. [2002], 'Gravitational collapse: The role of general relativity (reprinted from rivista del nuovo cimento, numero speziale, i, vol 257, 1969)', General Relativity and Gravitation 34(7), 1141-1165.

[13] Press, W. H. and Teukolsky, S. A. [1973], 'Perturbations of a rotating black hole. ii. dynamical stability of the kerr metric', The Astrophysical Journal 185, 649-674.

[14] Teukolsky, S. A. [1973], 'Perturbations of a rotating black hole. 1. fundamental equations for gravitational electromagnetic and neutrino field perturbations', Astrophys. J. 185, 635-647.

[15] Vilenkin, A. [1978], 'Exponential amplification of waves in the gravitational field of ultrarelativistic rotating body', Physics Letters B 78(2-3), 301-303.

[16] Zel'dovich, Y. B. [1972], 'Pis' ma zh. eksp. teor. fiz. 14, 270 (1971)[jetp lett. 14, 180 (1971)]', Zh. Eksp. Teor. Fiz 62 , 2076. 